\title{
A Herschel Spectroscopic Survey of Warm Molecular Gas in Local Infrared Luminous Galaxies
}

\author{
N. Lu ${ }^{1, *}$, Y. Zhao ${ }^{1,2}$, C. K. Xu ${ }^{1}$, Y. Gao ${ }^{2}$ and the GOALS FTS Team \\ ${ }^{1}$ NHSC/IPAC, MS 100-22, California Institute of Technology, Pasadena, CA 91125, USA \\ ${ }^{2}$ Purple Mountain Observatory, Chinese Academic of Sciences, Nanjing 210008, China \\ *email: lu@ipac.caltech.edu
}

\begin{abstract}
We describe an on-going 194-671 $\mu \mathrm{m}$ spectroscopic survey of a flux-limited sample of 125 local luminous infrared galaxies (LIRGs) with Herschel SPIRE Fourier Transform Spectrometer (FTS). The survey targets primarily the CO spectral line energy distribution (SLED), from $\mathrm{J}=4-3$ up to $\mathrm{J}=13-12$, to probe dense and warm molecular gas that should play an intimate role in star formation and/or active galactic nuclear activities in these galaxies. The program is about $75 \%$ finished. At $\mathrm{S} / \mathrm{N}>5$, besides the $\mathrm{CO}$ lines, we also detected [N II] $205 \mu \mathrm{m}$ and [C I] $370 \mu \mathrm{m}\left({ }^{3} \mathrm{P}_{2}-{ }^{3} \mathrm{P}_{1}\right)$ lines in every target observed. In about half of the observed targets, we also detected $[\mathrm{C} \mathrm{I}] 609 \mu \mathrm{m}\left({ }^{3} \mathrm{P}_{1}-{ }^{3} \mathrm{P}_{0}\right)$.
\end{abstract}

Keywords. galaxies: ISM — galaxies: starburst — infrared: galaxies — infrared: ISM

Our sample is a flux-limited subset of the Great Observatories All-Sky LIRGs Survey sample (GOALS; Armus et al. 2009, PASP, 121, 559). Fig. 1 illustrates our SPIRE/FTS sample selection of 125 galaxies with $L_{\mathrm{IR}}>10^{11} L_{\odot}$ and an IR flux $F_{\mathrm{IR}}>6.5 \times$ $10^{-13} \mathrm{~W} \mathrm{~m}^{-2}$, of which 32 are covered by other existing SPIRE/FTS programs. Each of the remaining 93 targets, which represent more typical LIRGs, is being observed by us in a single telescope pointing that targets the nuclear position, with an integration time long enough to detect the $\mathrm{CO}(\mathrm{J}=6-5)$ line at $\mathrm{S} / \mathrm{N}>5$. Our SPIRE/FTS program should be completed by early 2013. Fig. 2 shows a a typical spectrum observed, with expected or detected lines marked.

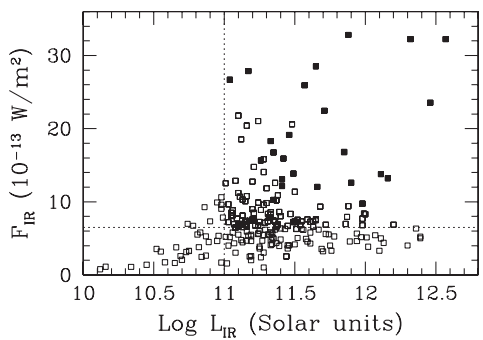

Figure 1. Plot of IR flux vs. IR luminosity for the full GOALS sample of 202 individual galaxies. The two dotted lines indicate the selection criteria for our SPIRE/FTS sample of 125 galaxies located in the right upper quadrant. The filled squares are 32 sample galaxies covered by other existing SPIRE/FTS programs.

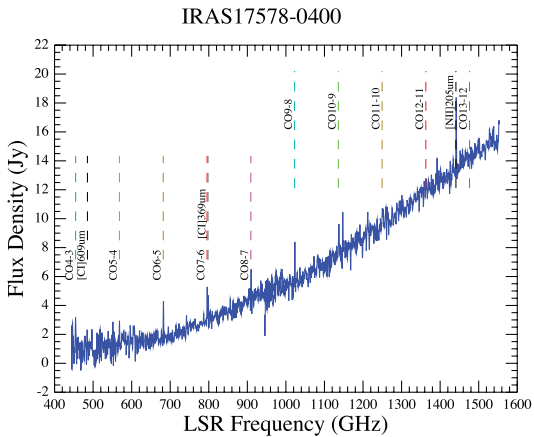

Figure 2. The observed SPIRE/FTS spectrum of IRAS 17578-0400 with the expected lines marked. 